Daimon. Revista Internacional de Filosofía, $n^{\circ} 83,2021$ pp. 105-121

ISSN: 1130-0507 (papel) y 1989-4651 (electrónico)

http://dx.doi.org/10.6018/daimon.368931

Las obras se publican en la edición electrónica de la revista bajo una licencia Creative Commons ReconocimientoNoComercial-SinObraDerivada 3.0 España (texto legal). Se pueden copiar, usar, difundir, transmitir y exponer públicamente, siempre que: i) se cite la autoría y la fuente original de su publicación (revista, editorial y URL de la obra); ii) no se usen para fines comerciales; iii) se mencione la existencia y especificaciones de esta licencia de uso. (c) (1)(-)(-)

\title{
El proto-evolucionismo ilustrado de la teoría del lenguaje de Adam Smith
}

\section{The enlightened proto-evolutionism of Adam Smith's theory of language}

JORGE LÓPEZ LLORET

\begin{abstract}
Resumen: El presente artículo analiza las importantes aportaciones de Adam Smith a la teoría del lenguaje. Identifica dos niveles argumentativos coordinados, el primero procedente de la lógica del análisis y la síntesis y el segundo procedente de una interpretación del lenguaje como una herramienta de adaptación y transformación social del entorno natural. Esto permite identificar una "hipótesis del desarrollo" del lenguaje, que evoluciona desde lo homogéneo desarticulado hasta lo heterogéneo articulado. A partir de aquí, se define la especificidad de la teoría del lenguaje de Smith entre los siglos XVIII y XIX, así como sus consecuencias estéticas y educativas.
\end{abstract}

Palabras Clave: Adam Smith, Lenguaje, Lógica, Gramática, Evolucionismo, Cultura.

\begin{abstract}
This article analyzes the important contributions of Adam Smith to the theory of language. It identifies two coordinated argumentative levels, the first coming from the logic of analysis and synthesis and the second coming from an interpretation of language as a tool for adaptation and social transformation of the natural environment. This allows identifying a language's "development hypothesis", which evolves from the disjointed homogeneous to the articulated heterogeneous. From here is defined the specificity of Smith's theory of language, between the eighteenth and nineteenth centuries, as well as its aesthetic and educational consequences.
\end{abstract}

Keywords: Adam Smith, Language, Logic, Grammar, Evolutionism, Culture.

\section{Introducción}

Robert C. Berwick y Noam Chomsky afirmaron en su estudio sobre la evolución y el lenguaje que "la indagación racional en la evolución de un sistema solo puede proseguir en la medida en que se entienda su naturaleza", pues de lo contrario sus manifestaciones nos parecerían caóticas (Berwick y Chomsky, 2016, 103). Creemos que es una buena introducción al programa lingüístico de la teoría del lenguaje de Adam Smith.

Recibido: 18/03/2019. Aceptado: 24/07/2019.

* Universidad de Sevilla. Profesor Titular de Universidad. Correo electrónico: lopezlloret@us.es. Líneas de investigación: Filosofía y Estética del Siglo XVIII; Retórica y Lenguaje en la Ilustración Escocesa; Estética y Cultura en la Teoría Evolucionista. Publicaciones recientes: López Lloret, J. (2019), «Adam Smith y la belleza de la ciencia», Anales del Seminario de Historia de la Filosofía, no 36 (1), 87-106; y López Lloret, J. (2019), «La metodología dramática en la obra de Adam Smith», Ágora. Papeles de Filosofía, n 38 (2), 71-93. 
Smith publicó sus Consideraciones sobre la formación original de los lenguajes en 1761 (a partir de ahora Consideraciones). ${ }^{1}$ Aunque esta obra está íntimamente conectada con sus Lecciones sobre Retórica, que suelen remitirse a los comienzos de su carrera como conferenciante en 1748 (Phillipson, 2011, 89), es posible que sus reflexiones sobre el lenguaje sean posteriores a 1755, dada su relación con el Diccionario de la lengua inglesa (1755) de Samuel Johnson. Este afirmó que al afrontar su tarea halló que el lenguaje inglés era rico y enérgico, pero carente de reglas (Johnson, 2006, 21), aunque optó por el poco sistemático orden alfabético. En la segunda mitad del siglo XVIII la necesidad de una reglamentación del lenguaje se dejó sentir con fuerza, multiplicándose notablemente en Gran Bretaña los diccionarios y gramáticas del idioma inglés (Miller, 1997, 39-40). Smith se esforzó para que de todo ello surgiera un proyecto unificado que integrara la estructura lógico-gramatical con la temporalidad del lenguaje hablado (Land, 1986, 131-2), algo evidente en su primera colaboración en la Edinburgh Review (1755), una reseña crítica del Diccionario de Johnson, cuya mayor debilidad, según Smith, residía en que su plan no era suficientemente gramatical, no subsumía los términos bajo clases generales y no jerarquizaba suficientemente los distintos significados (EPS, Edinburgh Review 1, 1).

Esta exigencia gramatical definió el programa de Consideraciones, que Smith consideró tan importante como para incorporarlo a la tercera edición (1767) de La teoría de los sentimientos morales, donde lo mantuvo hasta su muerte. En él Smith sistematizó el desarrollo del lenguaje desde el hipotético origen primitivo hasta el inglés moderno, reflexionando sobre la lógica y la motivación subyacentes a este proceso, lo que, unido a sus ideas sobre la historia expuestas en sus Lecciones sobre Jurisprudencia, generó una visión notablemente vanguardista. Tradicionalmente, hasta el siglo XVIII la cuestión del origen y diversificación de las lenguas se había afrontado en el marco de los textos bíblicos sobre el lenguaje adánico y la dispersión babélica. El grueso de las especulaciones sobre el lenguaje giraba, más bien, en torno a su relación con el pensamiento y la realidad, por una parte, y a su función comunicativa, por otra (Dascal, 1994, 15-51). Estos temas fueron recogidos y tratados históricamente por Rousseau y Condillac, cuyas obras Smith leyó inmediatamente (Dascal, 2006, 85). Por otra parte, Smith, directamente o a través de Condillac (Schreyer, 1978, 15-43), asumió la teoría lingüística de Mandeville, quien derivó el desarrollo del lenguaje humano a partir de nuestras necesidades animales. Finalmente, en el contexto de los estudios sobre la retórica se desarrolló la reflexión sobre la naturaleza lógica y gramatical del lenguaje, siendo referentes para Smith autores como Gabriel Girad

1 Al citar las obras de Adam Smith daremos en primer lugar la referencia a la Glasgow Edition, en la edición de la editorial Liberty. Como es norma, citaremos las Lectures on Rhetoric and Belles Lettres como LRBL, seguido del tomo y la página de los apuntes (en anverso o reverso), por ejemplo: LRBL, ii, 138; los Essays on Philosophical Subjects como EPS, seguido del ensayo, capítulo y párrafo, por ejemplo: EPS, Astronomy, IV, 7; las Lectures on Juriprudence como LJ, seguido del reporte que se trate, el número de volumen y la página de los apuntes, por ejemplo: LJ A, ii, 108; The Theory of Moral Sentiments como TMS, seguido del número de parte, sección, capítulo y párrafo, por ejemplo: TMS, II, iii, 2, 9; An Inquiry into the Nature and Causes of the Wealth of Nations como WN, seguido de número de libro, capítulo, parte (si la hubiera) y párrafo, por ejemplo: WN, I, xi, g, 21; The Correspondence of Adam Smith como Corr. seguido del número de carta; finalmente, citaremos Considerations Concerning the First Formation of Languages (que se haya en LRBL) como Languages, seguido del número de párrafo, por ejemplo, Languages, 43. Citaremos las traducciones siguiendo las normas de esta revista. 
o John Wilkins. La originalidad de Smith no reside en ninguno de estos temas sino en la integración del modelo histórico-hipotético sobre el origen del lenguaje, de Rousseau y Condillac, con el análisis comparativo de las lenguas históricas, esforzándose por ordenar los resultados en un proceso histórico coherente cuyas bases eran la lógica, la gramática y la precaria condición animal y social del ser humano. El resultado fue un lenguaje que no refleja la realidad, sino que la construye, un logro colectivo de naturaleza social.

Para mostrar esto último estructuraremos nuestro artículo en cuatro momentos expositivos y un epílogo. Primero estableceremos la lógica que, según Smith, subyace al desarrollo gramatical del lenguaje; en segundo lugar, expondremos los motivos por los que sería correcto calificarlo de proto-evolucionista; después identificaremos la hipótesis del desarrollo descrita en Consideraciones y, finalmente, su peculiar posición entre el evolucionismo decimonónico y la teoría ilustrada del desarrollo histórico. A modo de conclusión, aplicaremos al lenguaje la idea smithiana de las consecuencias no planificadas de nuestras decisiones, según la cual el lenguaje, originalmente un instrumento útil para la subsistencia personal en un entorno ingrato, acabó definiendo lenguajes colectivos con tradiciones poéticas estéticamente desinteresadas. A esto le subyace que se pueda seguir reclamando una educación culturalmente integral en las sociedades más avanzadas económicamente, donde el sujeto no ha de ser conformado meramente como una pieza del mecanismo que produce la riqueza de las naciones.

\section{2. "El mejor sistema de lógica en cualquier lenguaje"}

Dos años después de la aparición de Consideraciones Georg Baird envió a Smith el resumen de una obra que William Ward publicaría en 1765 con el título Ensayo sobre la gramática del idioma inglés. En su respuesta Smith no dijo mucho sobre este proyecto, pero expuso algunas ideas sobre el lenguaje, como la siguiente:

"Apruebo completamente su proyecto de una gramática racional y estoy convencido de que una obra de este tipo [...] no sería solo el mejor sistema de gramática, sino el mejor sistema de lógica en cualquier lenguaje, así como la mejor historia de los progresos naturales de la mente humana" (Corr., 69; traducción nuestra).

Tras esto Smith afirmó, algo enigmáticamente, que si "fuera a tratar el mismo tema intentaría comenzar con la consideración de los verbos, pues son, en mi opinión, las partes originales del lenguaje" (ibidem). Decimos "enigmáticamente" porque ya había publicado Consideraciones, donde no siguió ese plan, sino que comenzó con el estudio del sustantivo y no trató el verbo hasta el parágrafo 27 (más o menos como en la Lección III sobre retórica, impartida un mes y medio antes de la carta a Baird), afirmando solo que los verbos fueron "contemporáneos de los intentos iniciales de formación del lenguaje" (Languages, 27; Smith, 2018, 71). Esto suscita la duda sobre si habría que empezar con el nombre, como hizo de hecho Smith, o con el verbo, como propuso a Baird. ${ }^{2}$

2 El proceso se halla bien recogido en Dascal, 2006, 88-97. 
En su historia del lenguaje Smith recurrió a los procesos mentales de abstracción, basado en la síntesis (desarrollo a partir del nombre), y concreción, basado en el análisis (desarrollo a partir del verbo) (Land, 1986, 135-59; Christie, 1987, 206-25). Con esto afrontaba un problema que Rousseau consideró irresoluble, a saber, cómo era posible dar cuenta del desarrollo de unos términos abstractos que requerían un grado de experiencia del que carecían los primitivos y un nivel de pensamiento imposible sin un lenguaje complejo (Rousseau, 1989, 140 y 144-5). ${ }^{3}$ Smith respondió articulando una génesis basada en la interacción de los procesos sintéticos con los analíticos en un desarrollo a partir del nombre propio y del verbo impersonal.

Comenzó con el desarrollo a partir del nombre (Languages, 1-26; Smith, 2018, 39-71; Plank, 1992, 25-9). Partiendo de una conjetura formulada por Condillac (Condillac, 1999, 151-4), ${ }^{4}$ afirmó que cuando dos salvajes se encontraron por primera vez "tratarían de establecer signos que denotaran los objetos de su entorno más usuales y que más les interesaban" (LRBL, i, 17; traducción nuestra). El primer tipo de expresión lingüística pudo ser, pues, el nombre propio que denotaba un objeto particular (Languages, 1; Smith, 2018, 39). Tras esto se inició un camino de generalización, basado en la asociación de ideas resaltada por Hume (Hume, 1988, 54-8), que acabó produciendo los nombres comunes. Así, cuando el primitivo vio un árbol le asignó la palabra "árbol”, pero cuando observó otros árboles su semejanza hizo que los conectara con el primero y que les aplicara su nombre, recurriendo espontáneamente a la figura retórica conocida como "antonomasia" (Languages, 1; Smith, $2018,43) .{ }^{5}$ Esto generó un proceso de abstracción creciente por síntesis asociativa.

No obstante, con el nombre común era imposible saber a qué ejemplar se refería el signo "árbol". Para solucionar esto surgieron los adjetivos y las preposiciones (Languages, 3-7; Smith, 2018, 45-50), es decir, las cualidades y las relaciones que permitieron especificar los objetos, algo imposible solo con el nombre común. Esto, que en última instancia provenía de Aristóteles (Aristóteles, 1982, 34-42, 47-54 y 55-63), supone un proceso analítico de fragmentación del objeto en sus cualidades, relaciones, etcétera, pero también otro sintético, por el que una pluralidad de, por ejemplo, colores (en la manzana, el árbol, etcétera) se identifican como "verdes" y se diferencian de los "no verdes". La posibilidad del lenguaje exige, pues, en el caso del desarrollo a partir del nombre, la simultaneidad del proceso sintético de generalización y del proceso analítico de diferenciación categorial.

Con respecto al desarrollo a partir del verbo, Smith se basó en la sugerencia de Rousseau de que las primeras palabras expresaban una proposición completa, pues los eventos son en sí mismos realidades unificadas (Rousseau, 1989, 142; Dascal, 2006, 85-6). Para Smith estas primeras palabras fueron verbos impersonales porque en la naturaleza el agente y la acción no se diferencian (Languages, 28; Smith, 2018, 73), pero cuando se quiso distinguir un agente de otro el verbo impersonal, por un proceso analítico, se dividió en nombre y verbo personal y este, por un proceso de síntesis, se pudo aplicar a acciones similares de otros sujetos. El desarrollo a partir del verbo también fue, pues, un proceso

3 Smith se refirió a Rousseau en su carta a la Edinburgh Review de 1756, en la Lección III sobre retórica y en Consideraciones (EPS, Edinburgh Review 2, 11-17; LRBL, i, 19; Languages, 2; Smith, 1998, 220-4; Smith, 2018, 44).

4 Smith poseía un ejemplar de esta obra (Mizuta, 2000, 399). No proporcionamos el número de página, sino de catálogo.

5 Smith seguía a Du Marsais (Du Marsais, 2011, 76; Mizuta, 552). 
simultáneo de análisis y síntesis. Por lo tanto, aunque la lógica temporal global sería, a grandes rasgos: $1^{\circ}$ ) verbo impersonal, $2^{\circ}$ ) nombre propio más verbo personal, y $3^{\circ}$ ) nombre común más adjetivo/preposición, creemos más relevante que resaltar el proceso fue, tal y como Smith lo describió, de análisis creciente y, simultáneamente, síntesis creciente (es lo que denominaremos "hipótesis del desarrollo").

En su carta a Baird Smith se refirió a la gramática como "el mejor sistema de lógica". Era la lógica que se había ido definiendo en el complejo panorama británico de los siglos XVI a XVIII, donde el aristotelismo convivió con el racionalismo de Port-Royal y el empirismo lockeano, como muestra el Compendio de lógica de Hutcheson (Hutcheson, 2006, 1-56). ${ }^{6}$ Smith comenzó su carrera docente en Glasgow en la cátedra de Lógica, de modo que conocería el interés de esta disciplina por los problemas relativos al método desde que Petrus Ramus se los adjudicó a la lógica a costa de la retórica, algo muy presente en la lógica británica (Howell, 1961, 146-72). Thomas Blundeville estableció el modelo en The Art of Logike (1599), donde diferenció entre los métodos compositivo, que era sintético, y resolutivo, que era analítico (Blundeville, 1969, 55-6), algo que desarrollaron Samuel Smith (Smith, 1617, 202-3), Robert Sanderson en 1615 (Sanderson, 1741, 165-6), John Prideaux (Prideaux, 1650, 91-2) o Henry Aldrich en 1691 (Aldrich, 1750, 100), de donde llegó a Hutcheson (Hutcheson, 2006, 54-6). El entorno de todas estas obras (salvo la última) fue Oxford, en cuyas bibliotecas Smith pasó varios años como becario Snell (Scott, 1937, 37-45).

El interés de Smith por estas metodologías se puede rastrear en la Lección XXIV sobre retórica (LRBL, ii, 125-137), donde trató sobre los textos didácticos sin considerar el uso del análisis como método de descubrimiento que, siguiendo a Descartes, habían descrito Antonie Arnauld y Pierre Nicole (Arnauld y Nicole, 2017, 773). ${ }^{7}$ Dejó de lado la distinción entre "invención” y "enseñanza”, ocupándose de la síntesis y el análisis como recursos expositivos de un lenguaje cotidiano que no era un instrumento de investigación, menos aún en la situación primitiva descrita por Smith, algo que resultó muy innovador cuando este lo usó para articular un proceso histórico que, partiendo de la epistemología humeana (Raphael, 1977, 23-38), fue alimentado por la gestión que la imaginación hizo de un entorno ingrato.

\section{3. "La mejor historia de los progresos naturales de la mente humana"}

Consideraciones y las Lecciones sobre Retórica son contemporáneas de las Lecciones sobre Jurisprudencia, lo que permite aclarar mejor la novedad de las ideas lingüísticas de Smith. Volvamos al desarrollo a partir del nombre, donde nuestro autor, refinando una idea de Condillac (Condillac, 1999, 207 y 219), afirmó que los primeros nombres propios

6 Sobre la conexión de Aristóteles con el empirismo británico véase Sgarbi, 2013, 8-11.

7 Smith poseía un ejemplar (Mizuta, 2000, 89). Conocería además la distinción lockeana entre un método para adquirir conocimiento y otro para comunicarlo (Locke, 1986, 600). Gran parte de las lógicas más populares en Gran Bretaña a mediados del siglo XVIII, como las de Jean Le Clerc, Jean-Pierre de Crousaz, Isaac Watts o William Duncan, en gran parte conocidas por Smith (Mizuta, 2000, 966 y 1763), partían de la lógica de Arnauld y Nicole, dedicando una parte al método en sus mismos términos (Howell, 1971, 299-361). Con todo, Smith se centró en los aspectos comunicativos, según Howell la principal novedad de su retórica (Howell, 1971, 547-9). 
se referirían a objetos del entorno que satisfacían necesidades, como "cueva" (necesidad de cobijo), "árbol" (necesidad de comida) o "fuente" (necesidad de bebida) (Languages, 1; Smith, 2018, 39-40).

Esta familiaridad de los objetos le condujo a una teoría del origen del lenguaje dependiente de la naturaleza circundante y de las carencias humanas que esta puede satisfacer, de manera que las primeras palabras significaban necesidades que el entorno podía satisfacer, más que objetos. Esto provenía de La fábula de las abejas de Bernard Mandeville (Mandeville, 1997, 556-561), aunque Smith evitó unir a estas necesidades la más obvia y comunicativa del intercambio sexual, que para Mandeville fue más primordial. El lenguaje permitió la identificación de las posibilidades que el entorno ofrecía a nuestra viabilidad como especie, siendo, pues, un instrumento de adaptación al medio ambiente a través de la colaboración social.

Con respecto al desarrollo a partir del verbo impersonal, Smith siguió a ilustrados franceses como Diderot o, especialmente, Rousseau, para quienes el primer lenguaje fue "el grito de la naturaleza" (Rousseau, 1989, 140-1). Puso como ejemplo "venit", que indicaría algo así como "se acerca el león", entendido como un único evento complejo. Cuando después hubo que diferenciar la aproximación de otras bestias, se usó la misma palabra añadiéndole un nombre, por ejemplo: "venit ursus" o "venit lupus", pasando desde ahí a "significar la aproximación de cualquier objeto terrible" (Languages, 29; Smith, 2018, 74-5). En cualquier caso, el primer verbo impersonal surgió del pánico ante una naturaleza en la que nos acosaban los depredadores, que conformaban un entorno ingrato del que, gracias al lenguaje, nos podíamos zafar. Este grito fue una alerta, es decir, un vínculo social colaborativo en una situación que Smith, en su Historia de la astronomía, calificó de "precaria" y "brutal" (EPS, Astronomy, III, 1; Smith, 1998, 59). En ese contexto el lenguaje fue un instrumento de supervivencia ante algo salvajemente inmediato que, gracias a los procesos de análisis y síntesis, introdujo distinciones entre los recursos y peligros, consolidando la adaptación al entorno y reforzando el vínculo social. De aquí surgió lo que suele denominarse "teoría del desarrollo histórico en cuatro estadios", que apareció por primera vez en las Lecciones sobre Jurisprudencia (Meek, 1981, 112-28), que se remontan al año 1749. En esa fecha Montesquieu publicó El espíritu de las leyes (Phillipson, 2011, 102; Pauchant, 2017, 49-74), donde remitió las leyes y las formas de gobierno a factores como el clima o la geografía, pero Smith se basó en la adaptación económica, a través del lenguaje, a las posibilidades del entorno, desarrollándose progresivamente la pauta caza-recolección, pastoreo, agricultura y comercio-industria. Este proceso de adaptación y explotación del entorno fue interactuando con un lenguaje cada vez más estructurado, hasta llegar al prolijo y preciso idioma inglés que hablaban los industriosos contemporáneos de Smith.

En las Lecciones sobre Jurisprudencia hay sugerencias adicionales que ayudan a explicar por qué para Smith la historia del lenguaje era también la de los progresos de la mente humana, especialmente la referencia a la condición natural del ser humano, que aúna la debilidad física con un ingenio y capacidad de mejora que nos condujeron a la posibilidad de hablar (LJ A, vi, 8; Smith, 1995, 380). Puso como ejemplo de lo que podía resultar de aquí la necesidad de crear entornos artificiales para nuestra delicada piel, desde la ropa a la arquitectura, concluyendo que "casi todas las artes se han inventado y mejorado para suplir 
las necesidades de comer, beber, vestirse y alojarse" (LJ A, vi, 16; Smith, 1995, 383). ${ }^{8}$ Las tecnologías para satisfacer estas necesidades, desde la época de la caza-recolección hasta la del comercio-industria, se desarrollaron progresivamente, primero adaptándose al medio ambiente y después modificándolo (LJ A, i, 27-35; Smith, 1995, 46-9; Pocock, 2006, 280-1; Jonsson, 2013, 129-141; Haakonssen, 2016, 56-61). La tecnología que inició este proceso fue el lenguaje, cuya estructura analítico-sintética se estableció como el modelo de las tecnologías posteriores, permitiendo la viabilidad humana como especie mundana y dando lugar, finalmente, a la sofisticada sociedad analizada en La riqueza de las naciones.

Después de su famosa exposición de la división del trabajo en la fabricación de alfileres en WN, Smith se preguntó por la causa subyacente a este fenómeno organizativo. Tras indicar que dependía de nuestra propensión al intercambio, afirmó que lo más probable era que fuese "la consecuencia de las facultades discursivas y del lenguaje" (WN, I, ii, 2; Smith, 1979). Continuó comparando a los humanos con otros animales, quienes pueden colaborar en una cacería, pero no desarrollar intercambios contractuales basados en el lenguaje. Esto podría deberse, seguía diciendo, a que estos se valen por sí mismos al alcanzar en breve tiempo la madurez, mientras que el crecimiento humano es lento y necesita de los demás, lo que exige el vínculo lingüístico. Esta versión final de un argumento que se formuló en la Lección sobre Jurisprudencia impartida un día después de la carta a Baird (LJ A, vi, 44-45; Smith, 1995, 395) tiene la interesante implicación de que el lenguaje es una peculiaridad del ser humano como animal que necesita adaptarse a su entorno. Smith no planteó la hipótesis de un humano sin lenguaje porque, ipso facto, la especie resultaría inviable, no pudiendo preexistir a un instrumento que la hace ser como es, es decir, una especie inteligente y débil que logró su éxito adaptativo gracias a la cooperación sociolingüística. Por eso, lo primero que hicieron los primitivos cuando Smith subió el telón de Consideraciones fue hablar.

\section{La hipótesis del desarrollo}

Al unir la lógica con la historia natural del ser humano, Smith elaboró en Consideraciones una "hipótesis del desarrollo" que sintonizaba bien con la segunda mitad del siglo XVIII, pero también anticipaba planteamientos posteriores. Así, en el texto se lee lo siguiente:

“[...] el género humano ha aprendido gradualmente a partir y dividir casi cualquier evento en un gran número de partes metafísicas, expresadas con los miembros diversos del habla y combinadas de forma variable en los diferentes lugares de cada frase y sentencia" (Languages, 30; Smith, 2018, 76).

Cuando el lenguaje, gracias a coordinación de la síntesis con el análisis, se desarrolló desde el grito original de alerta hasta los complejos constructos gramaticales modernos, pasó de ser algo homogéneo desarticulado a ser algo heterogéneo articulado. Según Smith fue algo "gradual e insensible" (Languages, 16; Smith, 2018, 61), lo que hace pensar en márgenes tem-

8 Recordemos que las primeras palabras fueron "árbol" (comer), "fuente" (beber) y "cueva" (alojarse) y "venit" (necesidad de huir y defenderse). 
porales largos. Aunque no aventuró una cifra, conocía la asombrosa ampliación temporal de la historia natural propuesta por Diderot, ${ }^{9}$ quien no tuvo problema en hablar de millones de años:

"[...] que el embrión formado por esos elementos ha pasado por una infinidad de organizaciones y de desarrollos; que ha tenido, por herencia, movimiento, sensación, ideas, pensamiento, reflexión, conciencia, sentimientos, pasiones, signos, sonidos articulados, una lengua, ciencias, y artes; que han transcurrido millones de años entre cada uno de esos desarrollos; que tal vez tenga aún que sufrir otros desarrollos y adoptar otros crecimientos" (Diderot, 1992, 143).

Esta hipótesis del desarrollo fue un argumento básico de la embriología epigenética, que Smith, al igual que Diderot, conocía, como mostró en su carta a la Edinburgh Review (EPS, Edinburgh Review 2, 8; Smith, 1998, 218-9). Otro elemento fundamental de esta teoría, también destacado por Diderot, fue el gradualismo del desarrollo (aplicado por Smith al lenguaje), argumentable por la ampliación de los límites temporales. El entorno inmediato de Smith colaboró definitivamente en esta ampliación, pues uno de sus íntimos, James Hutton (editor de sus Ensayos filosóficos), introdujo el tiempo profundo en la geología con su Teoría de la tierra de 1788 (Toulmin y Goodfield, 1965, 155-9; Jackson, 2006, 89-92; Rudwick, 2014, 68-73), ${ }^{10}$ mientras que otro amigo, su cirujano John Hunter, ${ }^{11}$ elaboró una de las teorías proto-evolucionistas más sofisticadas de la segunda mitad del siglo XVIII (Dempster, 2005, 13-38; Moore, 2006, 482-99). Al final de Consideraciones, en una importante comparación del lenguaje con las máquinas, Smith sintonizaba bien con este complejo contexto:

“[...] el lenguaje ha llegado a ser más simple en sus rudimentos y principios en proporción al crecimiento en complejidad de su composición, sucediendo con él lo mismo que pasa comúnmente con las máquinas, todas las cuales, por lo general, son muy complejas en sus principios cuando son inventadas, habiendo a menudo un principio motor para cada uno de los movimientos que deben realizar. Quienes las mejoran observan que se puede aplicar un principio para producir varios de los movimientos, con lo que la máquina llega a ser gradualmente más simple, produciendo sus efectos con menos ruedas y principios motores. De la misma manera, en el lenguaje cada caso de cada sustantivo y cada tiempo de cada verbo fue expresado originalmente con una palabra diferente que solo servía para este propósito y no para otro. Sin embargo, la observación posterior descubrió que un conjunto de palabras era capaz de ocupar el lugar de aquel número infinito, de modo que cuatro o cinco preposiciones y media docena de verbos auxiliares podían responder al mismo fin que todas las declinaciones y conjugaciones de los lenguajes antiguos" (Languages, 41; Smith, 2018, 92-93).

9 Poseía un ejemplar de los Pensamientos sobre la interpretación de la naturaleza (Mizuta, 2000, 502).

10 Hutton presentó el primer esquema de su teoría (cuyas primeras investigaciones se remitían a los años cincuenta) en 1785 ante la Royal Society de Edimburgo. Smith estuvo presente (Repcheck, 2004, 142-6).

11 Smith asistió a las clases sobre anatomía de su hermano William en 1773 (Ross, 1995, 251). 
Esta comparación del lenguaje con la máquina fue relevante porque interpretó el desarrollo como un proceso de organización creciente, posible gracias a una articulación sintáctica cada vez más compleja y, paradójicamente, fácil de gestionar. No fue un proceso automático, pues la máquina lingüística fue un instrumento con el que el ser humano transformó la realidad a medida que se adaptaba a ella. No fue, por eso, un espejo progresivamente preciso del mundo o de la mente, sino algo "completamente artificial" que los rearticulaba (Languages, 28; Smith, 2018, 73). Así como el lenguaje no reflejaba la estructura de la realidad no humana, tampoco el sujeto era una entidad pasiva que recibía el mundo mediante sus sentidos, sino un agente que con el lenguaje hacía que la realidad no humana, homogénea y desestructurada, llegara a ser para él algo heterogéneo y estructurado. El sentido progresivo de este desarrollo no se debía a ningún determinismo natural o histórico, sino a que cada sociedad conducía el análisis y la síntesis un paso más allá de las que la antecedieron, desde el grito primitivo de alerta hasta el inglés moderno, una compleja combinación de griego, latín, francés y sajón que hizo posible una visión del mundo como la de Newton (Languages, 37-40; Smith, 2018, 89-92).

Fue un proceso creativo, lo que explica que Smith usara una metáfora escénica para referirse a la naturaleza como una suerte de teatro de la ópera, más allá de cuyo escenario no podíamos ir (EPS, Astronomy, II, 9; Smith, 1998, 54), ${ }^{12}$ y que, junto a esto, le preocupara desentrañar la función de las tres unidades clásicas de la tragedia, que trató como unidades de interés (equivalente a la unidad clásica de acción), tiempo y espacio (LRBL, ii, 81-89). Este proceso lo desencadenó la imaginación, que se sentía aterrada ante la homogeneidad desestructurada de la realidad no humana y solo se tranquilizó cuando, gracias al lenguaje, la realidad se fue haciendo más heterogénea y estructurada (Griswold, 2006, 46-54). En la construcción de una tragedia la imaginación transciende estos temores porque puede conformar su objeto a su medida, pudiéndose comprobar mejor en este caso la manera en la que funciona. Así, cuando un dramaturgo rompe la unidad de tiempo sucede lo siguiente:

"[...] nos intranquiliza el desconocer lo que ha sucedido durante dicho intervalo, dentro del cual deben haber ocurrido muchas cosas importantes, de las cuales no sabemos nada. Es decir, damos un salto desde un momento a otro, ignorando lo que los conecta" (LRBL, ii, 86-87; traducción nuestra).

La imaginación humana, como muestra el ejemplo del drama, se satisface cuando accede a muchos datos (análisis) unificados de una manera coherente y completa (síntesis). De la misma manera afronta la realidad no humana, como mostró Smith en su Historia de la astronomía:

"La filosofía es la ciencia de los principios conectivos de la naturaleza [...] en la naturaleza parecen proliferar los hechos solitarios e incoherentes con todo lo que

12 Esta metáfora, utilizada por Fontenelle para justificar el sistema del mundo de Descartes (Fontenelle, 1963, 43), fue retomada por Smith, Hume y Reid en clave epistemológicamente escéptica (Hume, 2010, 17; Reid, 2004, 250). 
los precede, y que por ende perturban el movimiento cómodo del pensamiento; que hacen que sus ideas procedan en saltos y corcovos irregulares, por así decirlo; y que tiende de esta manera a introducir las confusiones y desórdenes ya mencionados [...] La filosofía, al exponer las cadenas invisibles que conectan todos estos objetos dislocados, pretende traer el orden a este caos de apariencias discordes y chirriantes, apaciguar el tumulto en la imaginación y restaurar en ella [...] el tono de tranquilidad y compostura que le es al mismo tiempo más grato de por sí y más conforme a su naturaleza" (EPS, Astronomy, II, 12; Smith, 1998, 57).

La naturaleza se nos da como algo desordenado y desconectado y la ciencia surge porque nuestra imaginación desea el orden y la conexión. En este caso no construimos completamente el producto, pues los materiales naturales no son como los de un drama, ya que la apertura temporal de la naturaleza no humana siempre proporciona nuevos hechos que deben identificarse y sintetizarse con los ya asumidos. La imaginación trabaja aumentando el número de fenómenos específicos (análisis) e integrándolos, a la vez, en tramas cada vez más unificadas (síntesis) (Montes, 2016, 47-9). El ejemplo por excelencia de esto es el sistema del mundo de Newton, en el que lo que comenzó con un número reducido de fenómenos no unificados (la época del mito) acabó asumiendo un número impresionante de fenómenos bajo un único principio de síntesis, la gravitación universal (EPS, Astronomy, III, 1-2 y IV, 76; Smith, 1998, 60-1 y 111).

Todo eso fue posible porque se desarrolló el lenguaje y, básicamente, procedió según la pauta establecida por este. Antes de que surgieran el mito y la ciencia el ser humano ya utilizaba el lenguaje, aplicando los procedimientos de análisis y síntesis en la puesta a punto, a lo largo de la historia, de un instrumento que le permitía satisfacer colectivamente sus necesidades y afrontar las demandas con las que lo acosaba su entorno.

\section{Smith entre la Ilustración y el proto-evolucionismo}

En el planteamiento lingüístico de Smith Dios fue el gran ausente, ${ }^{13}$ no habiendo al comienzo nada más allá del hambre, la sed, el desamparo y el miedo. Esto lo distanció de las propuestas del siglo XVIII y lo aproximó a planteamientos evolucionistas del siglo XIX. Herbert Spencer, por ejemplo, elaboró una teoría similar a la suya en La filosofía del estilo (1852). Aunque no citó Consideraciones, se apoyó en contemporáneos y seguidores de Smith, como Lord Kames o Blair (Spencer, 1878, 10, 15 y 20), comenzando su proyecto donde terminó el de Smith, es decir, comparando el lenguaje con la máquina según un principio de adaptación funcionalista a la ley del mínimo esfuerzo:

“Considerando el lenguaje como un aparato de símbolos para la transmisión del pensamiento, podemos decir que le sucede como a cualquier aparato mecánico, a saber,

13 Las observaciones de Kennedy (2016, 473-482) referentes a La teoría de los sentimientos morales son pertinentes aquí. Peter Harrison $(2010,88)$ se preguntaba cómo podría funcionar el sistema de Smith sin una deidad, pero lo cierto es que en Consideraciones funciona sin ella. 
que mientras más simples sean y mejor articuladas estén sus partes, mayor será el efecto que produzcan" (Spencer, 1878, 11).

Al final de esta obra Spencer presentó un esquema general del desarrollo del lenguaje que era el mismo que propuso Smith:

“[...] en el pasado lejano los hombres solo tenían nombres y verbos para transmitir sus ideas [...] desde entonces hasta el presente el desarrollo se ha producido hacia un número mayor de implementos del pensamiento y, por ello, hacia una complejidad y variedad mayores en sus combinaciones [...] actualmente, usamos las sentencias como el hombre primitivo usaba las palabras [...] de mantenerse el proceso que hasta ahora se ha desarrollado, se producirá una heterogeneidad creciente en nuestros modos de expresión" (Spencer, 1878, 47).

A continuación, identificó este desarrollo lingüístico con los procesos naturales, que también proceden de lo simplemente yuxtapuesto a lo altamente organizado (Spencer, 1878, 48), idea que posteriormente aplicó a las sociedades en Los primeros principios, donde formuló su "ley de la evolución", según la cual esta era "el paso de una homogeneidad incoherente a una heterogeneidad coherente" (Spencer, 2008, 236). Esta conexión con Spencer es significativa porque fue uno de los referentes de lo que se conoce como "darwinismo social", que aplicó al desarrollo social el principio de la "supervivencia del más apto" (Spencer, 1864, 444; Harris, 1996, 106-11), un argumento básico en la defensa del libre mercado durante el siglo XIX, que buscaba apoyarse en una ciencia, la economía, cuya objetividad fuera comparable con la de otras, como la biología. En este contexto, La riqueza de las naciones se interpretaba como la primera defensa sólida del libre mercado y como la primera obra en la que la economía se presentaba como una ciencia objetiva, inspirada en el modelo fisicomatemático de Newton (Harris, 1996, 108; Hühn, 2017, 2-5).

Esta conexión con Spencer muestra el potencial histórico de conceder más importancia a la adaptación al entorno a través de la satisfacción colectiva de las necesidades que a un don divino para transmitir ideas. No obstante, Consideraciones presenta elementos característicos de un ilustrado. En el contexto positivista del siglo XIX los procesos analizados por la ciencia tendían a ser afrontados como algo que nos sucede, pero para Smith el lenguaje (y, a partir de él, el sistema de la economía) era algo que hacíamos, una tecnología cultural que en parte controlábamos y en parte se nos iba de las manos. ${ }^{14}$

El lenguaje no era para Smith algo denotativo sino constructivo. Si se compara su teoría histórica del lenguaje con, por ejemplo, la de John Locke, quedará más claro. Para este el lenguaje era un conjunto de signos homólogo a otro conjunto de signos, las ideas, que respondían a la realidad no humana. Dicho conjunto de signos se hallaba al final del proceso epistémico y debía reflejar con claridad el mundo de ideas previo (Locke, 1986, 394-6). Pero en el caso de Smith el lenguaje estaba al principio y las ideas surgían con él. ${ }^{15}$ Así, cuando analizó la definición progresiva de las partes de la oración a partir del grito primitivo, afirmó:

14 Tomamos la expresión "cultural technology" de Duncan, 1998, 41.

15 Según Schliesser, para Smith "el lenguaje y la mente se codesarrollan” (Schliesser, 2011, 18). 
“[...] la división de este evento en dos partes es completamente artificial, un efecto de la imperfección del lenguaje que, en esta ocasión como en muchas otras, suple con un número de palabras la falta de una que pudiera expresar de una vez la totalidad del hecho" (Languages, 28; Smith, 2018, 73).

El resultado es que el lenguaje fragmenta y reconecta artificialmente la realidad, según las necesidades de la imaginación humana, de manera que cuando accedemos a momentos más evolucionados de la sociedad (como la fase comercial) o del pensamiento (como la astronomía newtoniana), la realidad sobre la que operamos o que investigamos ya es un mundo articulado por el lenguaje. La economía, que es el resultado de nuestra tendencia a intercambiar cosas que, a su vez, surge de nuestra condición de animal que habla, es una realidad que, como el lenguaje que usamos, estamos construyendo a cada momento. Tampoco es algo que nos pasa, sino algo que hacemos, pues al generar un lenguaje que nos permitió afrontar nuestras necesidades y miedos, adaptándonos al entorno, también adaptamos progresivamente el entorno a nuestras necesidades y miedos.

Todo esto permite llegar a unas conclusiones que se apartan tanto de la conexión de Smith con un mercado inevitable en el que los beneficios se producen a partir de la confrontación entre individuos y empresas mejor o peor adaptados, como de la visión decimonónica de la evolución como una desesperante lucha de todos contra todos por sobrevivir. Pensamos en el tema de las consecuencias impremeditadas de nuestras decisiones y acciones, es decir, en que lo que hacemos puede conducir a cosas que no pretendíamos hacer, aunque podemos aprovecharlas.

\section{Conclusión: consecuencias impremeditadas}

Refiriéndose al lento desarrollo del lenguaje, Smith afirmó que sucedió "sin intención ni previsión por parte de los que establecieron el ejemplo originalmente, quienes nunca trataron de crear una regla general" (Languages, 16; Smith, 2018, 61). Se trata, según J. R. Otteson, de la primera aparición en su obra del principio de las consecuencias no planificadas de la acción humana (Otteson, 2013, 119-22), cuya expresión más conocida fue la idea de que el egoísmo privado podía generar beneficios públicos, algo que Smith vinculó con nuestra condición lingüística (WN, II, ii, 2; Smith, 1979, 16-7). En este caso lo negativo (el egoísmo) generaba lo positivo (el beneficio), aunque podría ser de otra manera, como sucedió con la división del trabajo y sus consecuencias negativas sobre los obreros sometidos a ella (WN, V, i, f, 50-55; Smith, 1979, 687-90) o con la pérdida progresiva de la plasticidad del lenguaje a medida que evolucionó, algo que Smith vio con nostalgia (Languages, 42-45; Smith, 2018, 94-101). Esto último nos conduce a una parte poco estudiada de su pensamiento, la conversión de los medios en fines, valorada por él como una de sus aportaciones más originales a la estética, pues rebasaba el utilitarismo de Hume:

"Nadie ha reparado hasta hoy, que yo sepa [...] que el ajuste preciso de los medios para alcanzar cualquier comodidad o disfrute es frecuentemente más apreciado que 
esa misma comodidad o placer cuya consecución parecía agotar todo su mérito" (TMS, IV, 1, 3; Smith, 1997, 326). ${ }^{16}$

En las Lecciones sobre Jurisprudencia explicó que esto se debía a nuestra condición de animales a la vez débiles e inteligentes, pues la necesidad constante de mejorar nuestras condiciones de vida acabó conduciéndonos a valorar cosas que no "dan una ventaja superior al cubrir las carencias de la naturaleza" (LJ A, vi, 13; Smith, 1995, 381), como sucedió con el establecimiento de las formas poéticas del lenguaje. Al afrontar la delicadeza de nuestra constitución los medios llegaron a importarnos tanto, afirmó Smith, que incluso "el color, la más débil y superficial de todas las distinciones, se convierte en objeto de" consideración (ibidem), algo, evidentemente, aplicable al sonido. En Consideraciones explicó el desarrollo del sistema de las concordancias lingüísticas recurriendo a "aquel amor a la similitud de sonido y aquel deleite en el retorno de las mismas sílabas, que es el fundamento de la analogía en todos los lenguajes" (Languages, 10; Smith, 2018, 54), y en la Lección XXI sobre retórica se preguntó por el sentido del verso, respondiendo que "esta forma es mucho más difícil que la prosa, lo que muestra claramente que la finalidad principal del poeta fue la distracción y el entretenimiento" (LRBL, ii, 76 y 116). Era un caso evidente de complacencia en los medios formales que nos pone a las puertas del desinterés kantiano. ${ }^{17} \mathrm{El}$ lenguaje seguía siendo el instrumento adaptativo fundamental en la transformación económica del entorno propiciada por nuestra tendencia constante a persuadir, pero acabó desarrollando tradiciones literarias y poéticas como las que Smith analizó en sus Lecciones sobre Retórica. Es decir, al final nos encontramos ante el orgullo cívico de pertenecer a una comunidad cuyo lenguaje tiene la capacidad de producir belleza, algo que Smith asumió de clásicos como Isócrates, quien afirmó en "Sobre el cambio de fortunas":

"Con las demás cualidades que tenemos [...] en nada aventajamos a los animales, sino que incluso somos inferiores a la mayoría de ellos en rapidez, fuerza y otros recursos. Pero como existe en nosotros la posibilidad de convencernos mutuamente y de aclararnos aquello sobre lo que tomamos decisiones, no solo nos libramos de la vida salvaje, sino que nos reunimos, habitamos ciudades, establecimos leyes, descubrimos las técnicas y de todo cuanto hemos inventado la palabra es la que ayudó a establecerlo. Ella determinó con leyes lo que es justo e injusto, lo bello y lo vergonzoso" (Isócrates, 1980, 140). ${ }^{18}$

La teoría del lenguaje de Smith nos permite ir más allá de la imagen popular que se tiene de él y de su obra, basada sobre todo en la interpretación de La riqueza de las naciones como el retrato de un mundo pragmático en el que los individuos y las empresas se autorregulan compitiendo por lograr el mayor beneficio posible (Sheehan y Wahrman, 2015, 264-9; Mayr, 1986, 171-80). En este marco, hemos llegado a pensar que la educación debe ser ante todo la formación de profesionales capacitados para seguir alimentando ese mundo, interpretado

16 El utilitarismo de Hume en Hume, 1988, 416-22 y Hume, 1991, 79-100.

17 Del primer momento del juicio de gusto (Kant, 2012, 260).

18 Smith poseía varias ediciones de las obras de Isócrates (Mizuta, 2000, 864-866). 
como algo que nos pasa y a lo que hemos de adaptarnos, no como algo que hacemos. Pero nuestro autor no pensaba del todo así. Él se acercó a la economía por lo mismo que a la ética, la literatura, el arte o el lenguaje, porque le interesaba el ser humano. Smith, quien era un humanista que se enorgullecía de su dominio del griego y el latín y de su biblioteca personal, nutridísima de ediciones originales y traducciones de los clásicos grecolatinos, consideraría discutible la educación tan desequilibrada que actualmente potenciamos. De hecho, pensaba que la educación para paliar aquella formación tan unilateral era uno de los ámbitos en los que se podría tolerar el intervencionismo estatal, sobre todo en lo que se refiere a la lectura y la escritura (WN, V, i, f, 51-52; Smith, 1979, 689-70; Quinn, 2013, 120-9). Después de todo, el lenguaje, esa estructura adaptativa que acabó conduciéndonos a la economía del comercio y la industria, llegó a ser algo más, algo que dotó de valor humano a la riqueza de las naciones porque abría la posibilidad para todos de una relación humana desinteresada, de la libre celebración de la vida comunitaria en la cultura.

\section{Bibliografía citada}

Aldrich, H. (1750), Artis Logicae Compendium, Oxford: J. Fletcher.

Aristóteles (1982), Tratados de lógica (Organon), Madrid: Gredos.

Arnauld, A. y Nicole, P. (2017), La lógica o el arte de pensar, Oviedo: KRK.

Berwick, R. C. y Chomsky, N. (2016), ¿Por qué solo nosotros? Evolución y lenguaje, Barcelona: Kairós.

Blundeville, T. (1969), The Art of Logike, Amsterdam: Da Capo Press.

Christie, J. R. R. (1987), «Adam Smith's metaphysics of language», en: A. E. Benjamin, G. N. Cantor y J. R. R. Christie (eds.): The Figural and the Literal, Manchester: Manchester University Press, pp. 202-29.

Condillac, E. B. de (1999), Ensayo sobre el origen de los conocimientos humanos, Madrid: Tecnos.

Dascal, M. (1994), «Lenguaje y conocimiento en la filosofía moderna», en: E. de Olaso (ed.): Enciclopedia Iberoamericana de Filosofía 6. Del Renacimiento a la Ilustración, Madrid: Trotta, pp. 15-51.

Dascal, M. (2006), «Adam Smith's Theory of Language», en: K. Haakonssen (ed.): The Cambridge Companion to Adam Smith, Cambridge: Cambridge University Press, pp. 79-111.

Dempster, W. J. (2005), The Illustrious Hunter and the Darwins, Sussex: The Book Guild.

Diderot, D. (1992), Sobre la interpretación de la naturaleza, Barcelona: Anthropos.

Du Marsais, C. Ch. (2011), Des Tropes, París: Manucius.

Duncan, I. (1998), «Adam Smith, Samuel Johnson and the institutions of English», en: R. Crawford (ed.): The Scottish Invention of English Literature, Cambridge: Cambridge University Press, pp. 37-54.

Fontenelle, B. le B. (1963) Conversaciones sobre la pluralidad de los mundos, Madrid: Aguilar.

Griswold, Ch. L. (2006), «Imagination: Morals, Science, and Arts», en: K. Haakonssen (ed.): The Cambridge Companion to Adam Smith, Cambridge: Cambridge University Press, pp. 22-56. 
Haakonssen, K. (2016), «The Lectures on Jurisprudence», en: R. P. Hanley (ed.): Adam Smith. His Life, Thought, and Legacy, Princeton: Princeton University Press, pp. 48-66. Harris, M. (1996), El desarrollo de la teoría antropológica. Historia de las teorías de la cultura, México: Siglo XXI.

Harrison, P. (2010), «Adam Smith, Natural Theology, and the Natural Sciences», en: P. Oslington (ed.): Adam Smith as Theologian, New York: Routledge, pp. 77-91.

Howell, W. S. (1961), Logic and Rhetoric in England, 1500-1700, Nueva York: Russell and Russell.

Howell, W. S. (1971), Eighteenth-Century British Logic and Rhetoric, Princeton/New Jersey, Princeton University Press.

Hühn, M. P. (2017), «Adam Smith's Philosophy of Science: Economics as Moral Imagination», Journal of Business Ethics, https://doi.org/10.1007/s10551-017-3548-9.

Hume, D. (1988), Tratado de la naturaleza humana, Madrid: Tecnos.

Hume, D. (1991), Investigación sobre los principios de la moral, Madrid: Espasa Calpe.

Hume, D. (2010), Historia natural de la religión, Madrid: Tecnos.

Hutcheson, F. (2006), Logic, Metaphysics, and the Natural Sociability of Mankind, Indianapolis: Liberty Fund.

Isócrates (1980), Discursos II, Madrid: Gredos.

Jackson, P. W. (2006), The Chronologers' Quest, Cambridge: Cambridge University Press.

Johnson, S. (2006), A Dictionary of the English Language: An Anthology, London: Penguin.

Jonsson, F. A. (2013), Enlightenment's Frontier. The Scottish Highlands and the Origins of Environmentalism, New Haven: Yale University Press.

Kant, I. (2012), Crítica del discernimiento, Madrid: Alianza.

Kennedy, G. (2016), «Adam Smith on religion», en: Ch. J. Berry, M. P. Paganelli y C. Smith (eds.), The Oxford Handbook of Adam Smith, Oxford: Oxford University Press, pp. 464-84.

Land, S. K. (1986), The Philosophy of Language in Britain. Major Theories from Hobbes to Thomas Reid, New York: AMS Press.

Locke, J. (1986), Ensayo sobre el entendimiento humano, México: FCE.

Mandeville, B. (1997), La fábula de las abejas, México: FCE.

Mayr, O. (1986), Autority, Liberty and Automatic Machinery in Early Modern Europe, Baltimore: The John Hopkins University Press.

Meek, R. L. (1981), Los orígenes de la ciencia social. El desarrollo de la teoría de los cuatro estadios, Madrid: Siglo XXI.

Miller, Th. P. (1997), The Formation of College English, Pittsburgh: University of Pittsburgh.

Mizuta, H. (2000), Adam Smith's Library. A Catalogue. Oxford: Clarendon Press.

Montes, L. (2016), «Newtonianism and Adam Smith», en: Ch Berry, M. P. Paganelli y C. Smith (eds.): The Oxford Handbook to Adam Smith, Oxford: Oxord University Press, pp. 36-53.

Moore, W. (2006), The Knife Man, Londres: Bantam Books.

Mossner, I. S. y Ross, I. S. (eds.) (1977), The Correspondence of Adam Smith, Oxford: Clarendon Press.

Otteson, J. R. (2013), Adam Smith, Nueva York: Bloomsbury. 
Pauchant, T. C. (2017), «Adam Smith's four stages theory of socio-cultural evolution: new insights from his 1749 lecture», The Adam Smith Review, n 9, pp. 49-74.

Phillipson, N. (2011), Adam Smith. An Enlightened Life, London: Penguin.

Plank, F. (1992), «Adam Smith: grammatical economist», en: P. Jones y A. S. Skinner (eds.): Adam Smith Reviewed, Edinburgh: Edinburgh University Press, pp. 21-55.

Pocock, J. G. A. (2006), «Adam Smith and History», en: K. Haakonssen (ed.): The Cambridge Companion to Adam Smith, Cambridge: Cambirdge University Press, pp. 270-87.

Prideaux, J. (1650), Hypomnemata Logica, Oxford, s.e.

Quinn, K. (2013), «Adam Smith on Education», Critical Review. A Journal of Politics and Society, $\mathrm{n}^{\circ}$ 25-1, pp. 120-9.

Raphael, D. D. (1977), «"The true old Humean philosophy" and its influence on Adam Smith», en: G. P. Morice (ed.): David Hume. Bicentenary Papers, Edinburgh: Edinburgh University Press, pp. 23-38.

Reid, Th. (2004), Investigación sobre la mente humana según los principios del sentido común, Madrid: Trotta.

Repcheck, J. (2004), The Man Who Found Time, London: Pocket Books.

Ross, I. S. (1995), The Life of Adam Smith, Oxford: Clarendon Press.

Rousseau, J.-J. (1989), Discurso sobre el origen y los fundamentos de la desigualdad entre los hombres y otros escritos, Madrid, Tecnos.

Rudwick, M. J. S. (2014), Earth Deep History, Chicago: The University of Chicago Press.

Sanderson, R. (1741), Logicae Artis Compendium, Oxford: E. Broughton.

Schliesser, E. (2011), «Reading Adam Smith after Darwin: On the evolution of propensities, institutions, and sentiments», Journal of Economic Behavior and Organization, $\mathrm{n}^{\circ} 77$, 14-22.

Schreyer, R. (1978), «Condillac, Mandeville, and the Origin of Language», Historiographia Linguistica, $\mathrm{n}^{\mathrm{o}}$ 5, 15-43.

Scott, W. R. (1937), Adam Smith as Student and Professor, Glasgow: Jackson, Son and Company.

Sgarbi, M. (2013), The Aristotelian Tradition and the Rise of British Empiricism, Heidelberg: Springer.

Sheehan, J. y Wahrman, D. (2015), Invisible Hands. Self-Organization and the Eighteenth Century, Chicago: The University of Chicago Press.

Smith, A. (1979), Investigación sobre la naturaleza y causas de la riqueza de las naciones, traducción de Gabriel Franco, México: Fondo de Cultura Económica.

Smith, A. (1981), An Inquiry into the Nature and Causes of the Wealth of Nations (1776), Indianapolis: Liberty Fund.

Smith, A. (1982a), Essays on Philosophical Subjects (1795), Indianapolis: Liberty Fund.

Smith, A. (1982b), Lectures on Jurisprudence, Indianapolis: Liberty Fund.

Smith, A. (1982c), The Theory of Moral Sentiments (1759), Indianapolis: Liberty Fund.

Smith, A. (1985), Lectures on Rhetoric and Belles Lettres, Indianapolis: Liberty Fund.

Smith, A. (1995), Lecciones sobre Jurisprudencia, traducción de Manuel Escamilla Castillo y José Joaquín Jiménez Sánchez, Granada: Comares.

Smith, A. (1997), La teoría de los sentimientos morales, traducción de Carlos Rodríguez Braun, Madrid: Alianza. 
Smith, A. (1998), Ensayos filosóficos, traducción de Carlos Rodríguez Braun, Madrid: Pirámide.

Smith, A. (2018), Consideraciones sobre la formación original de los lenguajes (1761), traducción de Jorge López Lloret, Oviedo: KRK.

Smith, S. (1617), Aditus ad Logicam, Oxford: Iosephus Barnesius.

Spencer, H. (1864), The Principles of Biology, Vol. I, London: Williams and Norgate.

Spencer, H. (1878), Philosophy of Style, Nueva York: Apleton and Company.

Spencer, H. (2008), Los primeros principios, Granada: Comares.

Toulmin, S. y Goodfield, J. (1965), The Discovery of Time, Chicago: The University of Chicago Press. 